\title{
A Collaborative Framework for QoC-driven Context Information Provision
}

\author{
Elarbi Badidi \\ College of Information Technology \\ United Arab Emirates University Al-Ain \\ United Arab Emirates
}

\begin{abstract}
Context-aware services need up-to-date context information to be able to adapt their behavior to current situations of mobile users. Context Information Services (CIS) typically provide infrastructure support for collection, management, and dissemination of context information concerning a number of subjects. Subjects may be users, objects such as handheld devices and equipment, or the environment of users. We propose, in this paper, a framework for context provisioning that relies on a federation of cloud-based context brokers. Context Brokers (CBs) of the federation cooperate to assist context consumers' select suitable context providers, which can fulfill their quality-of-context (QoC) requirements. They also negotiate Context Level Agreements (CLAs) with selected context providers on behalf of context consumers. We describe our auction-based algorithm for CISs selection together with the CLA negotiation protocol.
\end{abstract}

\section{Introduction}

With the proliferation of smartphones and Internet enabled mobile devices, personalization of services enables dynamic customization of content and delivered services that are relevant to the mobile user based on her current context and preferences. For example, the personalization may recommend to the mobile user a specific store in a city based on her location, preferences, and previous purchases. The quality of the recommendation is extremely dependent on the quality of context information (QoC). Several definitions of the concept of personalization have been proposed in the literature. We consider the definition formulated by Paul Hagen [1] as short and complete.

"Personalization is the ability to provide content and services tailored to individuals based on knowledge about their preferences and behavior"
It emerges from these definitions that the aim of personalization is improving the mobile user's experience by adapting applications and services as much as possible to her preferences and contexts. It involves: (1) Collecting information about the user, her preferences, current activities, and context information such as location and surrounding objects; (2) Anticipating her requirements by making recommendations for additional services; and (3) Building trust and loyalty with the mobile user so that he/she gets satisfaction in using the service [2].

Modern smartphones have several built-in sensors. In the Android platform, mobile application can access all of these sensors as local services through well-defined interfaces. With these advances in mobile devices technology, users are more and more considering smartphones as personal information-processing tools. They expect being able to execute any application on top of a mobile device. However, given the massive amount of context information to be collected from the user and the environment and then processed, mobile devices are still lacking the necessary resources, in terms of storage and computing power, compared with a conventional information-processing device such as a workstation or a laptop.

One way to overcome the limitations of local computing and storage resources and battery life in mobile devices is using mobile cloud computing [3]. It allows using the resources of a cloud computing platform - such as Microsoft Azure, Amazon EC2, and Google AppEngine. Therefore, large-sized applications could not be deployed on mobile devices. A well-matching solution is to deploy reusable services on the service provider side, and to let mobile devices access these services over the Internet and adapt the interface according to the current situation of the mobile user. Context information that supports such adaptation is typically obtained from a context management system, which collects, aggregates and supplies context information from various context information sources such as mobile devices and environment sensors. With the advent of service oriented computing, these context management systems have evolved to what is called Context Information Services. 
Given the massive amount of data that these services collect and store, there is a growing trend to deploy them on the cloud to take advantage of the benefits procured by the cloud deployment and delivery models. CISs as well as context-aware services, which require context information, are very often physically distributed. For instance, context sources providing information about the current temperature may be far from the applications that need to adapt their services to the prevailing weather conditions. It is likely that these CISs provide the same context information but with different QoC. Furthermore, finding suitable context providers is not enough, as the levels of QoC to be delivered and the terms and conditions of context delivery need to be negotiated with these providers.

In this paper, we describe our proposed framework for QoC-driven selection of CISs. The main component of the framework is a federation of Context Brokers (CBs), which are in charge of the selection of CISs and mediating between context consumers and CISs to reach agreements that explicitly describe expected context information and QoC levels.

The rest of this paper is organized as follows. Section 2 presents background information on context management systems, and the concepts of quality of context indicators and Context Levels Agreement. Section 3 describes related work and motivation for this work. Section 4 presents an overview of the proposed framework. Section 5 describes the QoC-driven algorithm for CISs' selection. Section 6 presents the CLA negotiation process. Finally, Section 7 concludes the paper.

\section{Background}

\subsection{Context management systems}

To adapt their behavior and service provisioning to the user's current situation and the changing environment, context-aware services, which are the context information consumers, have traditionally used one of the following two scenarios: (1) Obtaining raw context information from various context sources or directly from users and environment sensors, and aggregating raw data to obtain high-level context information, which is then used in making adaptation decisions; and (2) Obtaining high-level context information from a common context management system, which collects raw data from various context sources and aggregates that data.

Context management systems provide infrastructure support for collection, management, and dissemination of context information about a number of subjects. They acquire context information from numerous context sources, which are usually third parties that collect and provide context information. Subjects may be users, objects such as handheld devices and equipment, or the environment of users. Several works on context-awareness surveyed existing context management systems to understand their features and shortcomings. Baldauf et al. [4] provided a survey on many context-aware systems and compare them in terms of sensing support, context model, context processing, resource discovery, historical context data, security, and privacy.

With the advent of service orientation, these context management systems have evolved to what is called Context Information Services. Ebling et al. [5] developed a CIS, named Owl, which supports context-aware applications. Its architecture is extensible and flexible that it accommodates heterogeneous context sources. Moreover, It provides a programming model that permits to implement both synchronous queries and asynchronous event notifications. Lei et al. [6] designed and implemented a middleware system for context collection and dissemination as a CIS. They addressed the issues of extensibility of the CIS architecture to support heterogeneous context sources, privacy, and quality of context information. Schmidt et al. [7] designed and implemented a generic CIS that permits context collection, discovery and monitoring. The service provides a Web service interface that allows its integration in heterogeneous environments. Its implementation uses OWL to describe context information and SPARQL to query and monitor context information.

Because of the substantial amount of context information that CISs collect, process, and store, there a high trend toward deploying CISs on the cloud. Using the cloud infrastructure, a context provider can offer cheaper and more reliable CISs than is possible within its premises. Cloud-based CISs can utilize the full processing and storage resources of the cloud infrastructure if needed. They can scale up when additional compute and storage resources are required owing to a rise in the demand for context information. Conversely, they can scale down when the demand for context information decreases.

\subsection{Quality-of-context indicators}

Adaptive applications and CISs obtain raw context data from heterogeneous context sources, which use diverse kinds of sensors with different levels of reliability. Besides, the aggregation of raw context data, to generate high-level context information, might introduce additional biases. To deal with this reliability issue, context information has been characterized by some properties referred in literature as quality-of-context (QoC) indicators [8][9]. Krause et al. [9] defined the QoC as:

"Quality of Context (QoC) is any inherent information that describes context information and can be used to determine the worth of the information for a specific application. This includes information about the provisioning process the information has undergone ('history', "age"), but not estimations about future provisioning steps it might run through."

QoC greatly impacts the behavior of contextaware services. Context information with lower quality increases the risk of making incorrect contextbased adaptation decisions. The importance of QoC indicators for the adaptation process has been recognized in several works on context-awareness. Buchholz et al. [8] identified the following QoC indicators: precision, freshness, temporal resolution, 
spatial resolution, and probability of correctness. Precision represents the granularity with which context information describes a real world situation. Freshness denotes the time that elapses between the determination of context information and its delivery to a requester. Spatial resolution characterizes the precision with which the physical area, to which an instance of context information is applicable, is expressed. Temporal resolution is the period of time during which a single instance of context information is applicable. Probability of correctness signifies the probability that a piece of context information is correct. Kim et al. [10] added the following QoC indicators: accuracy, completeness, representation consistency, and access security. Neisse et al. [14] described their proposed QoC framework, which is based on three main QoC parameters, namely, up-todateness, precision, and resolution.

Few works investigated the issues of modeling and measuring QoC. Filho et al. [16] described a OWL-DL QoC model and methods for measuring QoC by taking into account the fact that context information might be modified after sensing and described into a high semantic level. Manzoor et al. [17] considered QoC to be composed of two components, QoC sources and QoC parameters. QoC sources denote information concerning the sources, which collect context information, the subjects about which context information is collected, and the environment where context information is sensed and collected.

\subsection{Context level agreements}

A Context Level Agreement (CLA) is an agreement between a context provider and a context consumer (adaptive application) about the guarantees of delivered context information. It describes common understandings and expectations between the two parties. The guarantees concern the context information and the QoC levels to be delivered.

The typical components of a CLA are:

- Parties: represents the parties involved in the CLA and their respective roles (context consumer and context provider).

- Activation time: represents the period of time at which the CLA will be valid.

- Scope: defines the types of context information covered in the agreement.

- Context-level objectives (CLOs): Represent the levels of QoC that both parties agree on, and habitually include a number of quality indicators such as accuracy and freshness.

- Penalties: specifies the penalties for not meeting the stated context level objectives, such as getting discount or having the right to terminate the contract in light of unsatisfactory context levels.

- Exclusions: specifies what is not covered in the CLA.

- Administration: defines the processes to assess the CLA objectives, and describes the responsibility of the context provider regarding the control of each of these processes.
A CLA life cycle includes five phases, which are: Development, Negotiation and Sales, Implementation, Execution, and Assessment.

\section{Related work and motivation}

Brokerage of context information has been the subject of several research efforts over the last few years. Chen et al. [11] described a context brokerbased architecture, dubbed $\mathrm{CoBrA}$, which provides support to pervasive context-aware systems. It uses the Web Ontology Language OWL to model context ontologies and offer support for context reasoning. Moreover, it enforces users privacy policies when sharing their context information. Reetz et al. [12] evaluated the performance of a context information provisioning system that relies on a context broker using black-box measurements and a simulation model. They identified major parameters and related models for the response delay of the main components of the system. Kiani et al. [13] defined a model of a large-scale system for context dissemination that relies on a federation of context brokers interconnected through a publish-subscribe model. The model addresses scalability and mobility issues faced in a single-broker system that uses synchronous communication between the system's components. They also introduced a mobile broker to facilitate the involvement of mobile devices in context provisioning and consumption efficiently.

Only few works in the area of context-awareness in pervasive environments have investigated the issue of CISs selection. Huebscher et al. [15] proposed an algorithm to rank context providers according to their QoC capabilities. They represent QoC indicators as a vector and use the Euclidean distance as a ranking metric for context providers. Neisse et al. [14] showed how their QoC model might be applied in a QoC management architecture to support contextaware service providers in the management of QoC and trustworthiness values, and to select trustworthy context providers.

Moreover, only a small number of research works in the area of context-awareness have investigated the issue of negotiation of context delivery based on the quality requirement of the context consumer. To the best of our knowledge, the most significant work that investigated the establishment and negotiation of CLAs is the work of Khedr et al. [16]. In this work, the authors described a multi-agent middleware, which uses a negotiation protocol, to facilitate the development of adaptive context-aware personalized applications, and an ontology model to represent context information. Our approach differs from Khedr's approach in that our our proposed framework relies on context brokers instead of agents to negotiate CLAs on behalf of context-consumers and a publish/subscribe model for monitoring QoC provisioning.

Our work aims at facilitating mediation between context consumers and context providers and providing support for automated selection of suitable CISs based on their QoC capabilities and the consumer's QoC requirements. Finding the right context offering is not an easy task for context consumers given the variety of context offerings. 
Moreover, dealing with a context provider requires knowledge of its operating environment, the availability of context management tools, and the service terms and conditions. Collecting this information for multiple context providers is likely to be an arduous task that is expensive and time consuming. Context Brokers with their know-how and value-added services will assist context consumers in: (a) finding appropriate context offerings, (b) negotiating the terms and conditions for context information delivery, and (c) offering a single interface to interact with multiple context providers.

With the growing adoption of cloud services and the deployment of services on the cloud as Softwareas-a-Service or Platform-as-a-service, we anticipate that context brokers will be deployed on the cloud to offer their services to a large base of context providers and context consumers. Gartner predicts that in parallel with the growing adoption of cloud services Cloud Brokers will emerge [18]. They will mainly be in charge of the management of the utilization, performance, and delivery of cloud services as well as intermediation between service consumer and multiple cloud providers.

\section{Framework overview}

Fig. 1 depicts our proposed framework for CISs' selection. It is founded on a federation of cloud-based context brokers. The research community has already recognized the need for cloud federation given the heterogeneity in cloud offerings [19][20]. A cloud federation extends the reach of both service providers and service consumers. A service is no longer accessible only to requestors of its cloud. Instead, requestors may request services across cloud borders (similar to the cellular network). The main components of the framework are: Context Consumers (CCs), Context Brokers (CBs), and
Context Providers (CPs). Context providers deploying their CISs on different clouds of the federation may provide similar or different CISs.

\subsection{Context-consumers}

Context-aware services (CAS) are the adaptive applications, which understand situational context and adapts their behavior according to the user's activity, location, and the changing circumstances in the user's environment. They are the consumers of context information obtained from context-providers. They are typically implemented as a Web service. A CAS can be responsive to various situational conditions, such as:

- The location of the user.

- The time at which the user invokes the service.

- The activity that the user is carrying out at the time it invokes the service.

- The preferences that the user may have defined prior to invoking the service.

- The device (smartphone, tablet, laptop, etc.) that the user is using to invoke the service.

\subsection{Context brokers' federation}

The Federation consists of context brokers, from several clouds, that cooperate to achieve intermediation between context consumers and CISs at a large scale. Federated routes connect disparate brokers across various clouds, while context consumers connect to brokers on their own cloud or found in a brokers' directory. Context brokers may share information about CISs and the kind of context information that these services can provide. If a context broker can not satisfy the request of a context consumer for specific context information, it forwards the request to other context brokers, which may have the knowledge about CISs providing the requested context information.

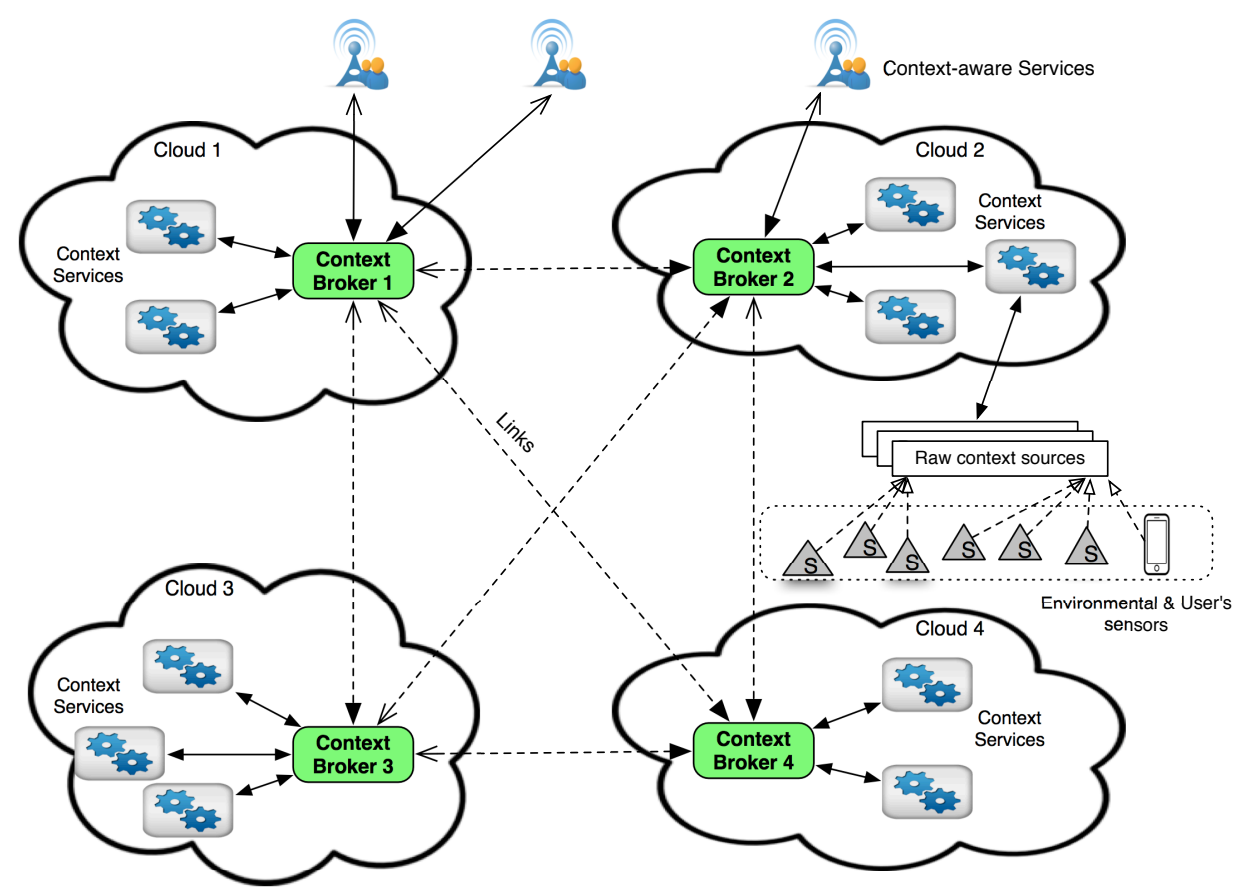

Figure 1. Context brokers federation 
Context brokers may be organized by geography, or context information type. Here are some use cases of the federation:

- Geography: context consumer's requests may be routed to a context broker close to the consumer.

- Context information type: high value context consumers may be routed to context brokers that have the knowledge on CISs providing requested context information type.

- Load balancing: interactions among brokers may be triggered dynamically to cope with changes in actual or anticipated load of brokers.

- High availability: routing may be changed to a new context broker if a solicited one becomes unavailable.

\subsection{Context brokers}

Context Brokers deployed on several clouds collaborate to provide services across cloud borders. By specifying the links between them the federation is created. A link contains information about the target broker, such as the reference of its Coordinator component. As we mentioned earlier, a Context Broker is a mediator service that decouples context consumers from context providers. Given that context consumers do not normally have the capabilities to negotiate, manage, and monitor QoC, they delegate management tasks, such as selection of appropriate context providers and Context Level Agreement (CLA) negotiation, to the Context Broker. The architecture of the Context Broker is depicted by figure 2. It includes several management operations, which cooperate to deliver personalized services to context consumers and context providers.

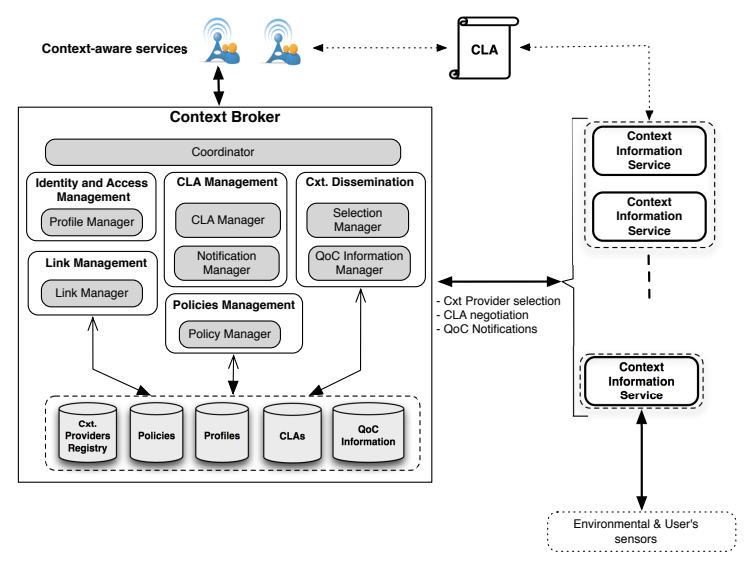

Figure 2. Context broker components

These operations are: Identity and Access Management (IAM), Policies Management (PM), CLA Management (CLAM), Context Dissemination (CD), Link Management (LM). These operations are controlled and coordinated by a Coordinator component. Back-end databases maintain information about providers' policies, consumers' profiles and preferences, CLAs, and dynamic QoC information.

The Selection Manager, which implements CD management operations, may implement various policies for the selection of suitable context providers, based on the consumer's QoC requirements and the context providers' QoC offerings. In next section, we describe a QoC-driven algorithm for the selection of cloud-based CISs.

The CLA Manager, which implements CLAM management operations, is in charge of carrying out the CLA negotiation process between the context consumer and the context provider selected by the Selection Manager.

The Notification Manager implements a topicbased publish-subscribe system in which contextproviders are the publishers and context-consumers are the subscribers.

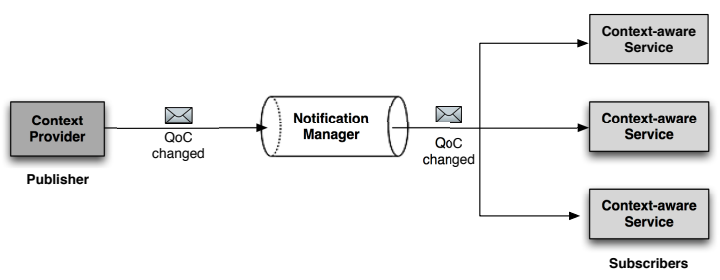

Figure 3. Topic-based publish/subscribe system

Fig. 3 depicts this model. QoC offerings of context information, requested by clients, represent the topics of the system. If there is a significant change in the current QoC of particular context information, the context-provider notifies the Notification Manager about the change in its QoC offering. Then, the Notification Manager notifies any subscriber to the corresponding QoC offering of that change. In addition to this model for getting QoC updates, the QoC Notification Manager also implements a regular on-demand request/response model.

The Profile Manager, which implements IAM management operations, is responsible for managing the profiles of context consumers, including their preferences in terms of context information types and required QoC. The Policy Manager, which implements PM management operations, is in charge of managing diverse kinds of policies such as authorization and access policies. The Link Manager component is responsible for managing links with other brokers of the federation. It provides the following link management operations: add_link(), remove link(), modify_link(), describe_link(), and list_links().

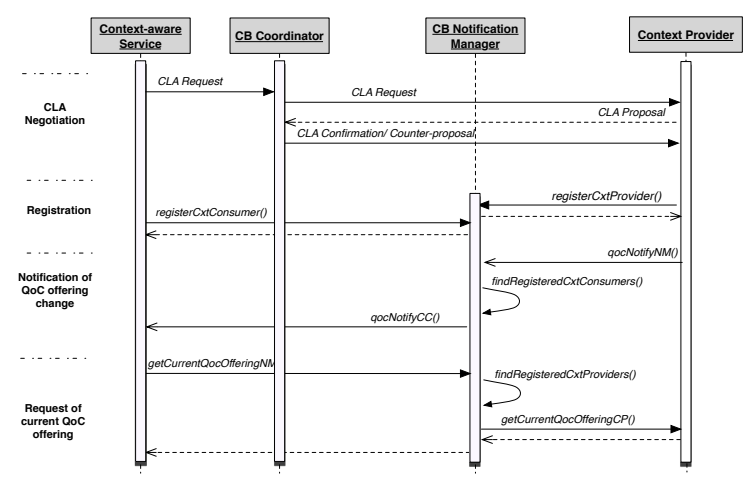

Figure 4. Typical interactions of the broker's components with context consumers and providers

Fig. 4 shows the typical interactions of the broker's components with the context consumer and context provider. The Notification Manager acts as an 
intermediary between publishers (context-providers) and subscribers (context-consumer) on a collection of context information (QoC offerings). Besides CLA negotiation, we distinguish three other kinds of interactions: registration with the Notification Manager, notification of QoC offering change, and request of current QoC offering.

\subsection{Context providers}

Context providers deploy their CISs, which may offer several types of context information such as location, temperature, and user activity. To determine their current QoC offering, context providers monitor and collect sensed data at selected measurement points. By aggregating collected data with its timestamps and data about sensors, a context provider can determine the current value of each QoC indicator for each category of context information.

The CLA Manager of the context provider is responsible for managing CLA templates, negotiating the CLA terms with the CB, or directly with context consumers, and implementing CLAs.

\section{QoC-based CIS selection}

In this section we describe our service selection algorithm that allows ranking the CISs in the federation with respect to the context consumer's required QoC, and then finding out the most suitable CIS in the federation to provide the context consumer with required context information and QoC. First, we describe how the algorithm works in the case of a single cloud. Then, we show how the selection is done at the level of the federation.

Let $C=\left\{C_{1}, C_{2}, \ldots, C_{m}\right\}$ represent the set of clouds of the federation $F$. One or several context brokers are deployed on each cloud of the federation. Let $B=\left\{B_{1}, B_{2}, \ldots, B_{l}\right\}$ be the list of Context Brokers of the federation $\mathrm{F}$.

\subsection{Single broker selection algorithm}

The context broker $B_{i}$ of cloud $C_{i}$ that receives the context-consumer request initiates an auction in order to get bids from relevant CISs that can handle the context-consumer request. We consider here the case of requests of just one kind of context information (e.g. location, humidity, temperature, etc.). Each bid is a vector, which describes the level of QoC for each of the quality indicators that the CIS may assure. This model corresponds to a multiple-items auction. After a predefined period, the broker $B_{i}$ closes the auction and ranks the bids according to the weight specified by the context-consumer for each quality indicator. It, then, selects the winner of the auction as the most suitable CIS to handle the context-consumer request. The auction models, proposed in the auction theory, are English auction, Sealed-Bid auction, Dutch auction, and Vickrey auction. As the bidders should not be aware of the bids of each other, the sealed-bid auction is the most relevant in our case. In the following, we describe the process of determining the winner of the auction.

Let $C S_{i}=\left\{C S_{1 i}, C S_{2 i}, \ldots, C S_{k i}\right\}$ be the set of CISs of the cloud $C_{i}$, with $1 \leq i \leq m$. We assume that QoC indicators are in normalized form with values between 0 and 1 . A value of 1 means highest quality and 0 means lowest quality. When submitting a request to $B_{i}$, the context consumer specifies her preferences with regard to the normalized QoC indicators that she can tolerate. Let $Q=\left\{Q_{1}, Q_{2}, \ldots\right.$, $\left.Q_{n}\right\}$ be the list of QoC indicators considered in the system. $B_{i}$ evaluates the utility function of each QoC attribute and the global utility function to determine whether the offer of a given CIS is acceptable or not. Several utility functions may be considered to evaluate the offers of CISs. In the following, we consider only two quality attributes, freshness and probability of correctness, to illustrate the approach. We define in equation (1) $U_{P}(p)$, the utility function for the probability of correctness quality attribute $p$. This function reaches its maximum value of 1 when $\mathrm{p}=1$ and decreases to 0 when $\mathrm{p}$ is decreasing to 0 .

$$
U_{P}(p)=\frac{p^{\beta_{P}}\left(1+\alpha_{P}\right)}{1+\alpha_{P} p^{\beta_{P}}}
$$

$p$ is the offer of the CIS for the probability of correctness and $\alpha_{P}$ is the desired value of the context consumer for $\mathrm{p} . \beta_{P}$ is a measure of the service consumer sensitivity to the probability of correctness quality attribute. When $\beta_{P}=0$, the service consumer is indifferent to the probability of correctness. When $\beta_{P}=1$, the service consumer is moderately sensitive to the probability of correctness. When $\beta_{P}>1$, the service consumer is increasingly sensitive to the probability of correctness. As $\beta_{P}$ increases, the service consumer is expressing increasing concern about it. For $\beta_{P}<1$, as $\mathrm{p}$ decreases to approach 0 , the service consumer is expressing increasing indifference to the probability of correctness. Fig. 3 shows $U_{P}(p)$ for $\alpha_{P}=0.75$, and $\beta_{P}=1,3$, and 5 respectively.

We consider also $U_{F}(f)$ the utility function for the freshness quality attribute $f$. It reaches its maximum, which is 1 , when $\mathrm{f}$ is 0 and decreases to 0 when $f$ reaches 1 as we are considering the normalized values.

$$
U_{F}(f)=\frac{1-f^{\beta_{F}}}{1+\alpha_{F} f^{\beta_{F}}}
$$

$f$ is the offer of the CIS for the freshness quality attribute and $\alpha_{F}$ is the desired value of the context consumer for that quality attribute. $\beta_{F}$ is a measure of the context consumer sensitivity to the freshness quality attribute similar to $\beta_{P}$. Fig. 4 shows $U_{F}(f)$ for $\beta_{F}=2$ and $\alpha_{F}=0.4,0.8$, and 0.95 respectively.

A global utility $U$ is a function of the individual utility functions $U_{j} .1 \leq j \leq n$

$$
U=f\left(U_{j}\right), 1 \leq j \leq n
$$

$U_{j}$ represents the individual utility function associated with the QoC attribute $Q_{j}$. If we assume that the QoC attributes are independent, the global utility function $U$ associated with the CIS $C S_{s i}$ can be expressed by the additive linear utility function as:

$$
U^{s}=w_{1} U_{1}^{s}+w_{2} U_{2}^{s}+\cdots+w_{n} U_{n}^{s}
$$

$w_{j}$ is the importance weight that the context consumer assigns to that attribute. Each weight is a number in the range $(0,1)$ and $\sum_{1}^{n} w_{j}=1$. The most suitable CIS, that we call target ${ }^{u}$, will be the one that maximizes the above additive utility.

Best offer $\leftarrow \max \left(U^{s}\right)$

Table 1 summarizes the steps of the algorithm. 
Table 1. Single broker selection algorithm

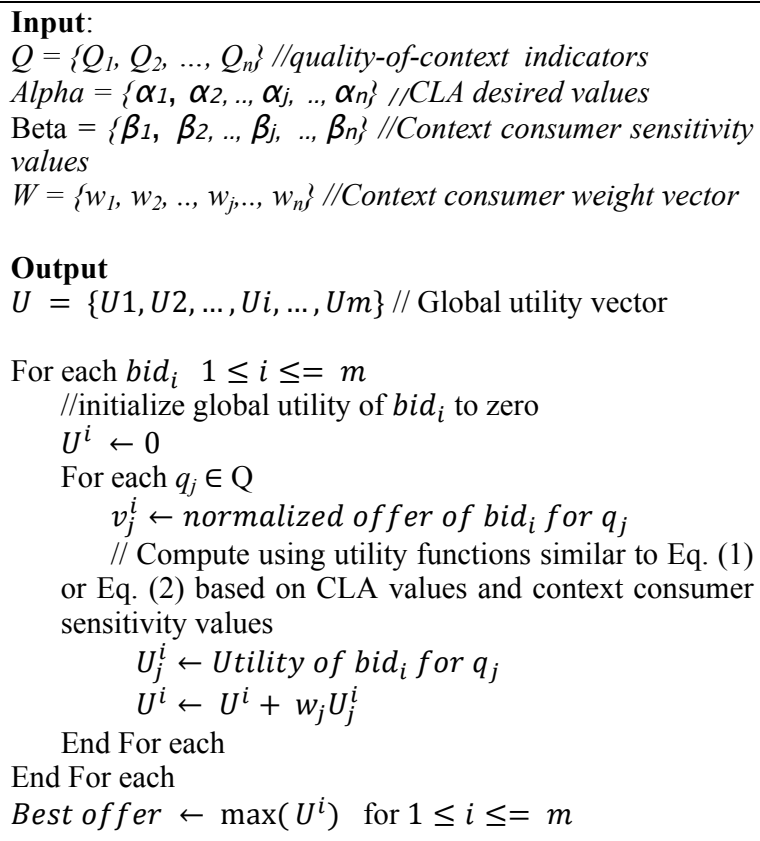

End For each

End For each

Best offer $\leftarrow \max \left(U^{i}\right)$ for $1 \leq i \leq=m$

As a proof of concept, we consider in Table 2 a scenario with CLA values for the probability of correctness and the freshness QoC indicators, the corresponding sensitivity parameters, and their associated weights. Table 3 depicts the offerings of 4 CISs for the setting of Table 2. Figure 5 plots the additive utility function for this scenario. It shows that the utility function reaches its maximal value when the freshness is small (close to 0) and the probability of correctness is high (close to 1 ).

Table 2. Example of context consumer parameters

\begin{tabular}{|l|c|c|}
\hline & Freshness & $\begin{array}{c}\text { Probability of } \\
\text { correctness }\end{array}$ \\
\hline CLA value & $\alpha_{F}=0.97$ & $\alpha_{P}=0.9$ \\
\hline Sensitivity & $\beta_{F}=4$ & $\beta_{P}=2$ \\
\hline Weight & $W_{F=} 0.6$ & $W_{P=} 0.4$ \\
\hline
\end{tabular}

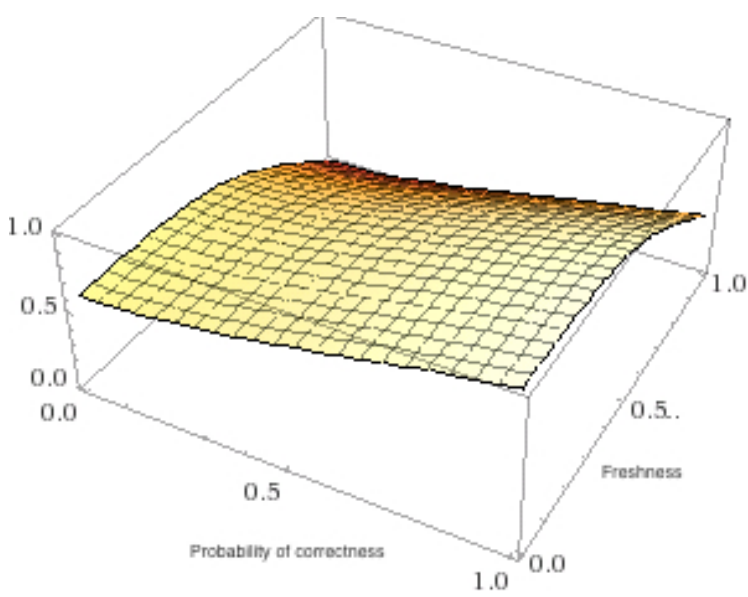

Figure 5. Utility function vs probability of correctness and freshness

The results in Table 3 show that the offer of $\mathrm{CIS}_{2}$ is the best CIS offer.
Table 3. CIS offers

\begin{tabular}{|c|c|c|c|c|c|}
\hline $\begin{array}{c}\text { Cloud } \\
\text { service }\end{array}$ & $\boldsymbol{f}$ & $\boldsymbol{p}$ & $\boldsymbol{U}_{\boldsymbol{F}}$ & $\boldsymbol{U}_{\boldsymbol{P}}$ & $\boldsymbol{U}$ \\
\hline CIS $_{\mathbf{1}}$ & 0.95 & 0.97 & 0.1036 & 0.9679 & 0.4494 \\
\hline CIS $_{\mathbf{2}}$ & 0.94 & 0.99 & 0.1247 & 0.9894 & 0.4706 \\
\hline CIS $_{3}$ & 0.96 & 0.98 & 0.0826 & 0.9787 & 0.4411 \\
\hline CIS $_{4}$ & 0.96 & 0.94 & 0.0826 & 0.9352 & 0.4236 \\
\hline
\end{tabular}

\subsection{Multiple brokers selection algorithm}

The previous subsection describes how the ranking of CISs is achieved within a single cloud. To find out the most suitable CIS within the federation, the initial context broker, which receives the contextconsumer request, forwards her QoC requirements to each broker of the federation. Each of these brokers executes, then, the selection algorithm to find, within their cloud, the best CIS capable of handling the context-consumer request. Selected CISs from the various clouds of the federation are then ranked to find out the best CIS, which maximizes the utility function expressed by (4).

\section{CLA negotiation}

Selection algorithms do not guarantee that the selected context offer is the best one. Therefore, to reach an agreement, the context consumer and the selected context provider need to negotiate the various quality levels of context information to be delivered. Fig. 6 depicts the CLA negotiation process. The steps of this process are as follows:

1) The context consumer submits a CLA request to the $\mathrm{CB}$ to find out an appropriate context provider that can provide required context information and fulfill its QoC requirements.

2) After authenticating the context consumer, the Coordinator requests its profile from the Profile Manager. Then, it requests from the Selection Manager to select a suitable context provider, which can deliver required context information according to the context consumer requirements.

3) The Coordinator requests policies of the selected context provider from the Policy Manager.

4) If the context consumer profile is available in the profile repository, for example, because the context consumer had previously used some services of the $\mathrm{CB}$, the Coordinator may determine whether the context providers, found by the Selection Manager, can handle or not the context consumer request. This decision relies on the profile of the context consumer and policies of the selected context providers.

5) If the context consumer's profile is not available in the profile repository, then the Coordinator asks the context consumer to provide information, such as preferences and desired levels of QoC, in order to create a new profile for the context consumer.

6) If at least one context provider can meet the context consumer requirements, the Coordinator requests from the CLA Manager to negotiate with that context provider the terms and conditions of context information delivery. 
7) The CB' CLA Manager forwards the CLA request, to the context provider's CLA Manager, requesting a proposal from the context provider. The context provider's CLA Manager parses the CLA request and validates it against its CLA templates.

8) If the CLA request is acceptable to the context provider, then its CLA Manager responds to the CLA request by sending back a CLA proposal. The CB analyses the CLA proposal to determine whether it meets all requirements of the context consumer.

9) If the context consumer's expectations can be met, then the $\mathrm{CB}$ accepts the offer of the context provider and sends a CLA confirmation to the context provider. Otherwise, it rejects the offer and makes a counter-proposal with different conditions, terms, costs, etc.

The operations of the CLA Manager of the context provider, with regards to CLA Negotiation, are as follows:

1) After receiving a CLA request, the CLA Manager analyses the CLA request.
2) If there is no issue with the structure of the CLA request, then the CLA Manager validates the CLA request against available CLA templates.

3) If the CLA request is acceptable, then the context provider's CLA Manager creates a CLA proposal and sends it to the $\mathrm{CB}$. At this point, the context provider will wait for CLA approval or dismissal from the $\mathrm{CB}$.

4) If the CLA request is not acceptable to the context provider, then its CLA Manager may suggest an alternative CLA to the CB and waits for acceptance or rejection.

5) Upon reception of the CLA approval from the $\mathrm{CB}$, the CLA Manager subscribes the context consumer in its registry and the CLA becomes ready for implementation.

In step 8 and step 9 of the above CLA negotiation process, a multi-attributes negotiation takes place between the CLA Managers of both parties. In each round, each party evaluates a utility function to determine if the offer (or counter-proposal) is acceptable or not. Utility functions similar to the ones we proposed in the previous section can be used.

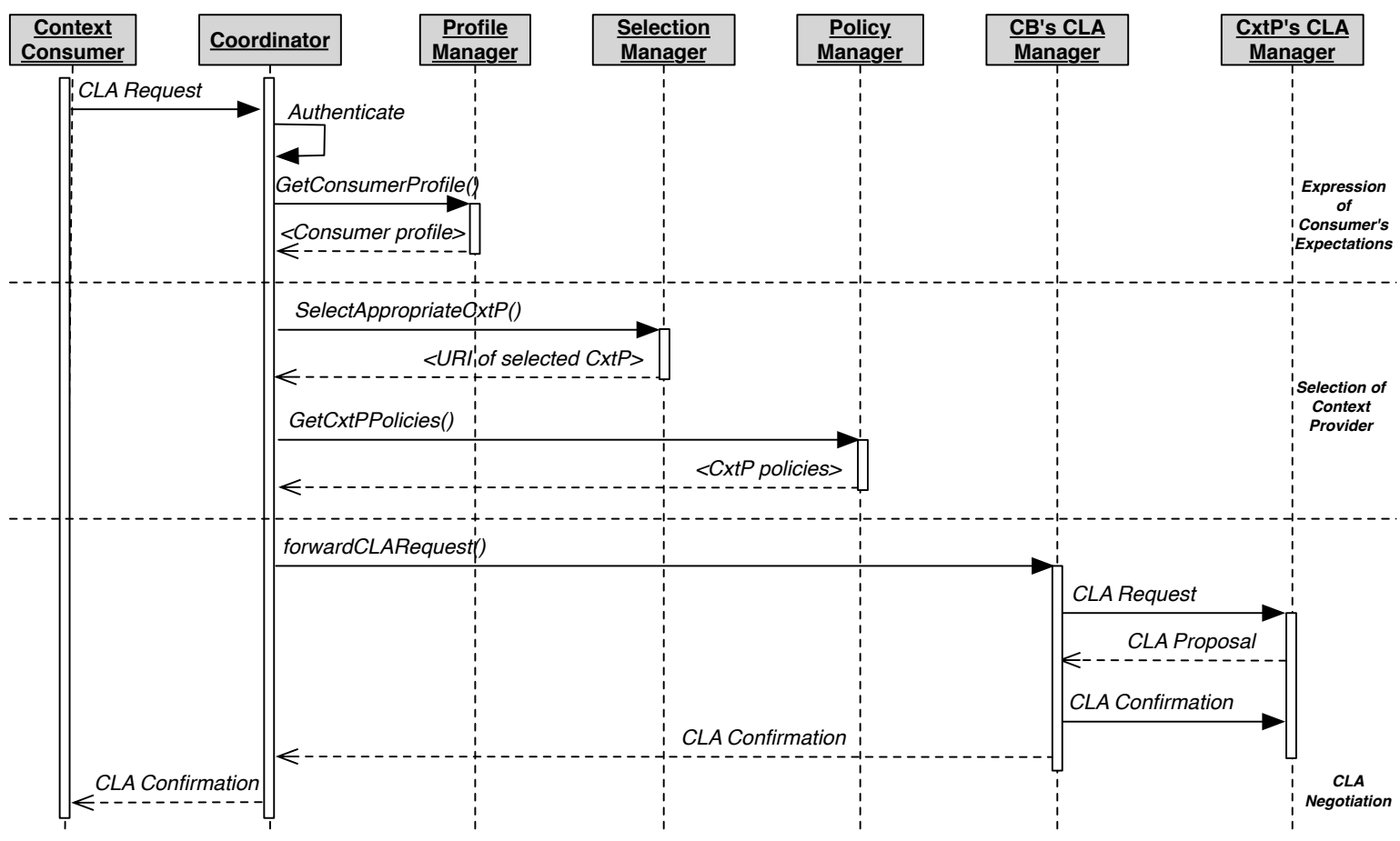

Figure 6. CLA negotiation protocol

\section{Conclusion}

Context-aware services, which provide adaptive and personalized services to mobile users, are facing the challenge of finding appropriate context providers that can supply them with relevant and high quality context information about numerous subjects. Given the colossal amount of context data collected, aggregated, and stored by Context Information Services (CIS), there is an increasing trend to deploy CISs on the cloud to take advantage of the cloud infrastructure and its delivery models.
In this paper, we have presented a framework for context information provision that relies on a federation of cloud-based context brokers. These brokers are in charge of selecting appropriate cloudbased CISs that can satisfy context consumers' requests for high-quality context information, and mediating between context consumers and selected CISs by carrying out, on behalf of context consumers, the negotiation of the terms and conditions of context delivery. The selection algorithm ranks the offers of potential CISs by evaluating a utility function for each individual offer. The best offer is the one that maximizes the utility function. To illustrate our 
approach, we have considered the weighted additive utility function of the individual utility functions of two quality-of-context attributes, freshness and probability of correctness.

\section{References}

[1] P. Hagen, with H. Manning and R. Souza. (1999) "Smart Personalization," Cambridge, MA: Forrester Research.

[2] G. Adomavicius and A. Tuzhilin (2005) "Personalization technologies: a process-oriented perspective," Commun. ACM, vol. 48, no. 10, pp. 83-90.

[3] H. T. Dinh, C. Lee, D. Niyato, and P. Wang (2011) "A survey of mobile cloud computing: architecture, applications, and approaches," Wirel. Commun. Mob. Comput., vol. 13, no. 18, pp. 1587-1611.

[4] M. Baldauf, S. Dustdar, and F. Rosenberg (2007) "A survey on context-aware systems," International Journal of Ad Hoc and Ubiquitous Computing, 2 (4), pp. 263-277.

[5] M. Ebling, G. D. Hunt, and H. Lei (2001) "Issues for context services for pervasive computing," Workshop on Middleware for Mobile Computing, Heidelberg.

[6] H. Lei, D. M. Sow, J. S. Davis, G. Banavar, and M.R. Ebling (2002) "The design and applications of a context service," Mob. Comput. Commun. Rev. 6(4), pp. 45-55.

[7] H. Schmidt, F. Flerlage, and F. J. Hauck (2004) “A generic context service for ubiquitous environments," In Proc. of the IEEE International Conference on Pervasive Computing and Communications (PERCOM), pp.1-6.

[8] T. Buchholz, A. Kpper, M. Schiffers (2003) "Quality of context: What it is and why we need it?," In Proc. of the 10th International Workshop of the HP OpenView University association (HPOVUA).

[9] M. Krause and I. Hochstatter (2005) "Challenges in modelling and using quality of context (QoC)," pp. 324-333.

[10] Y. Kim and K. Lee (2006) "A quality measurement method of context information in ubiquitous environments," in ICHIT'06. Washington, DC, USA: IEEE Computer Society, pp. 576-581.

[11] H. Chen, T. Finin, and A. Joshi (2004) "Semantic Web in the context broker architecture," In Proceedings of the Second IEEE Annual Conference on Pervasive Computing and Communications (PerCom 2004), pp. 277-286.

[12] E. S. Reetz, M. Knappmeyer, S.L. Kiani, N. Baker, and R. Tönjes (2012) "Performance evaluation of a context provisioning middleware," In Proc. of the International Conference on Computing, Networking and Communications (ICNC), pp. 441-445.

[13] S. L. Kiani, M. Knappmeyer, N. Baker, B. Moltchanov (2010) "A Federated Broker Architecture for Large Scale Context Dissemination," In Proc. of the IEEE 10th International Conference on Computer and Information Technology (CIT2010), pp. 2964-2969.

[14] R. Neisse, R., Wegdam, M., AND M. Van Sindren (2008) "Trustworthiness and quality of context information", In Proc. of the 9th International Conference for Young Computer Scientists (ICYCS'08). 1925-1931.

[15] C. Huebscher and A. Mccann (2005) "An adaptive middleware framework for context-aware applications", Personal Ubiquitous Comput., 10(1):12-20.

[16] J. B. Filho, A. D. Miron, and I. Satoh (2010) "Modeling and Measuring Quality of Context Information in Pervasive Environments," In Proc. of the IEEE International Conference on Advanced Information Networking and Applications, pp. 690-697.

[17] A. Manzoor, H. Truong, and S. Dustdar (2008) "On the evaluation of quality of context," In Proc. of the 3rd European Conference on Smart Sensing and Context (EuroSSC '08)

[18] Gartner (2009) "Gartner Says Cloud Consumers Need Brokerages to Unlock the Potential of Cloud Services,". http://www.gartner.com/newsroom/id/1064712
[19] D. Villegas, N. Bobroff, I. Rodero, J. Delgado, Y. Liu, A. Devarakonda, L. Fong, S. M. Sadjadi, and M. Parashar (2012) "Cloud federation in a layered service model," Journal of Computer and System Sciences, vol. 78, no. 5, pp. 1330-1344.

[20] A. N. Toosi, R. N. Calheiros, and R. Buyya (2014) "Interconnected Cloud Computing Environments: Challenges, Taxonomy, and Survey," ACM Computing Surveys (CSUR), vol. 47, no. 1, p. 7. 